\title{
Dual-band Planar Plasmonic Unidirectional Launching in a Semi-annular Apertures Array
}

\author{
Shan Huang ${ }^{*}{ }^{+,}{ }^{\ddagger}$, Chun-Yuan Wang ${ }^{\dagger}$, , Hung-Ying Chen ${ }^{\dagger}$, Meng-Hsien Lin ${ }^{\dagger}$, Yu-Jung Lu ${ }^{\dagger}$, , Shangjr Gwo*, ${ }^{{ }^{+},}$ \\ Supporting Information
}

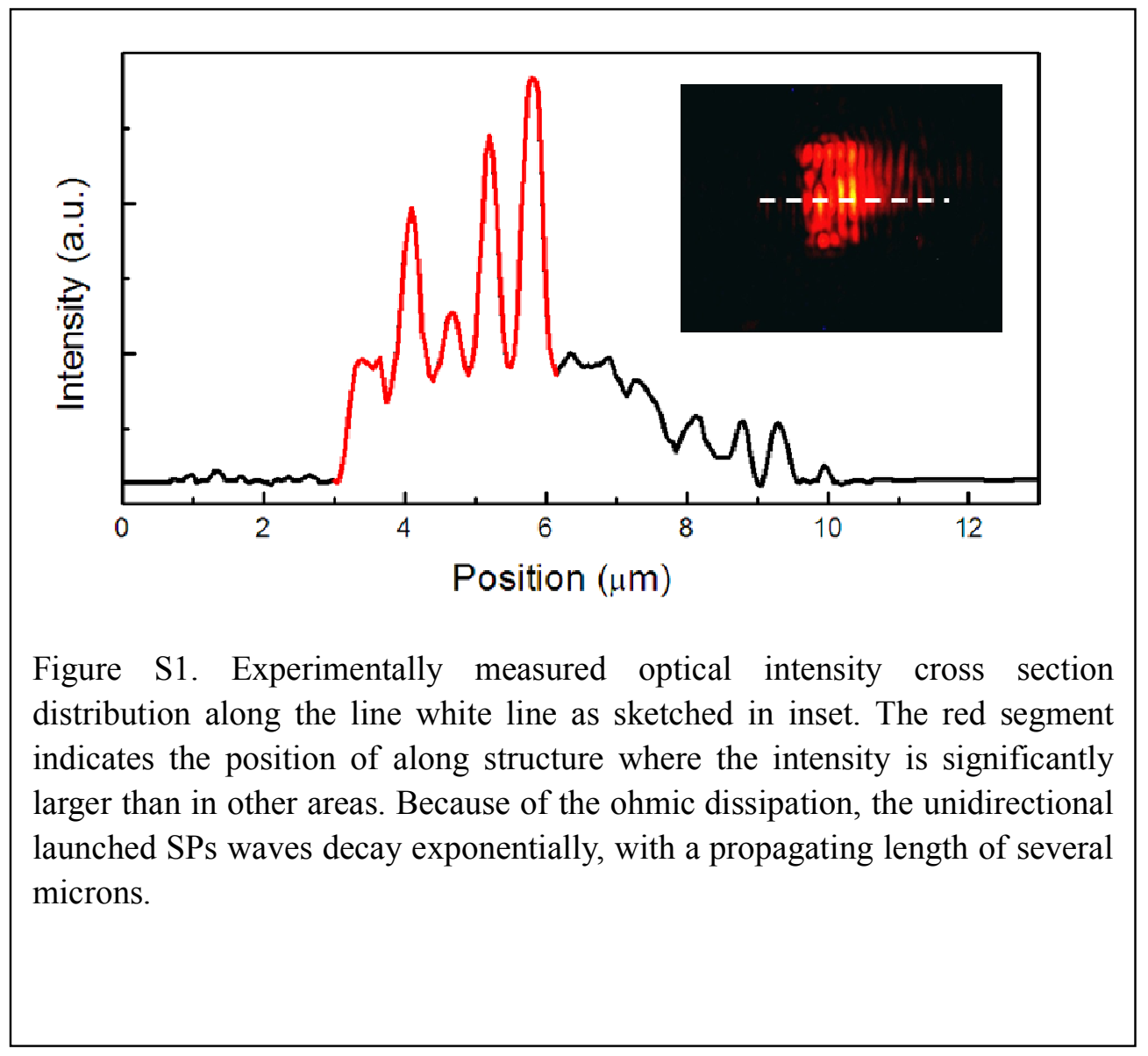




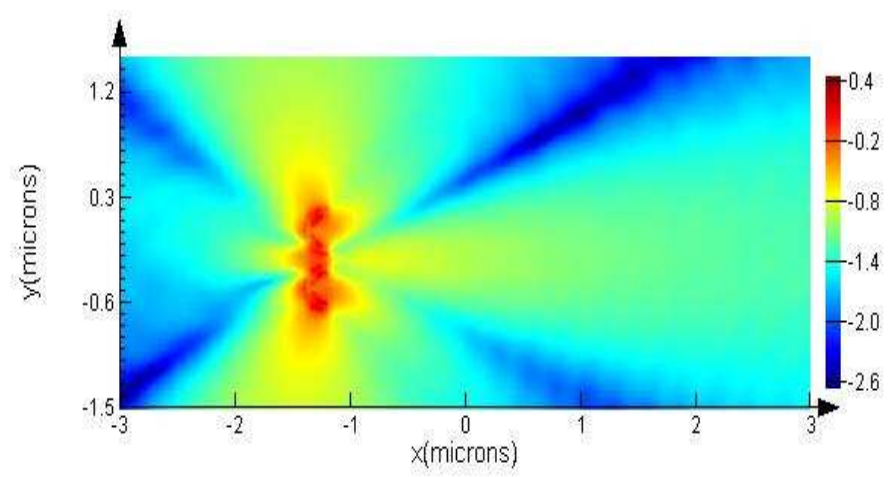

Figure S2. The FDTD simulated electric field magnitude distribution of two closed semi-annular apertures. The field distribution is no longer localized, and the SPs are asymmetrically scattered along the x-axis. Here, the inner and outer radii of the aperture are $r l=125 \mathrm{~nm}$ and $r 2=200 \mathrm{~nm}$, the gap between the two apertures is $90 \mathrm{~nm}$, and the incident wavelength is $588 \mathrm{~nm}$. The polarization is along $\mathrm{x}$ axis. 


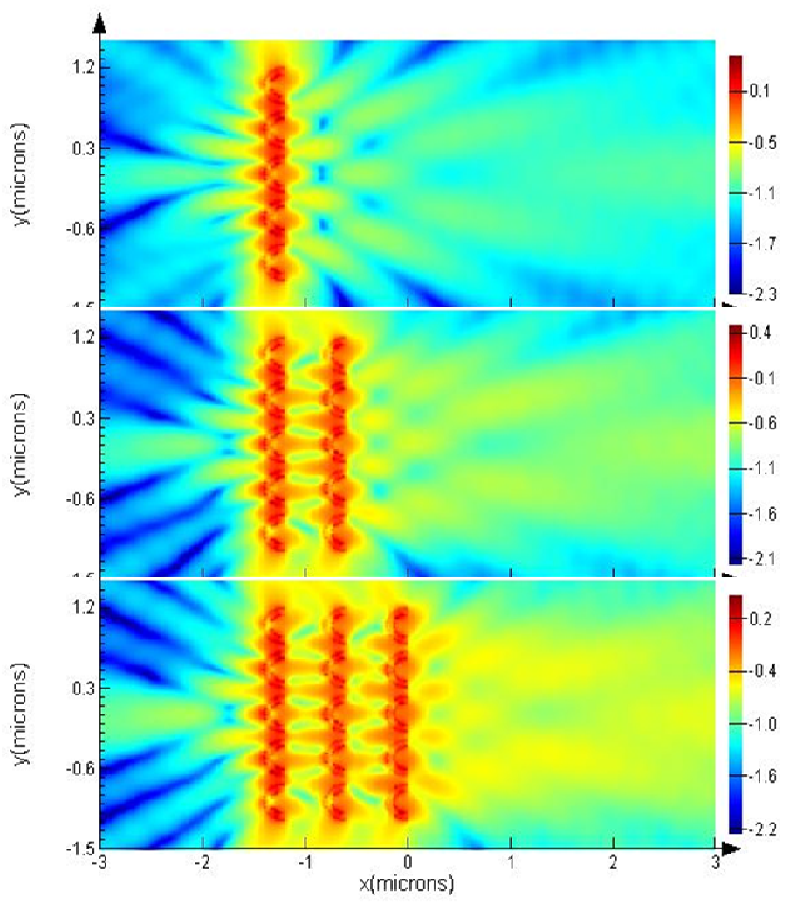

Figure S3. The FDTD simulated UL in one, two, and three semi-annular aperture chains. The UL becomes more apparent as the chain number increases. Here, the inner and outer radii of the aperture are $r l=125 \mathrm{~nm}$ and $r 2=200 \mathrm{~nm}$, the gap between the two apertures is $90 \mathrm{~nm}$, and the period of the chain is $600 \mathrm{~nm}$. The incident wavelength is $588 \mathrm{~nm}$, and the polarization is along the $\mathrm{x}$-axis. 
(a)

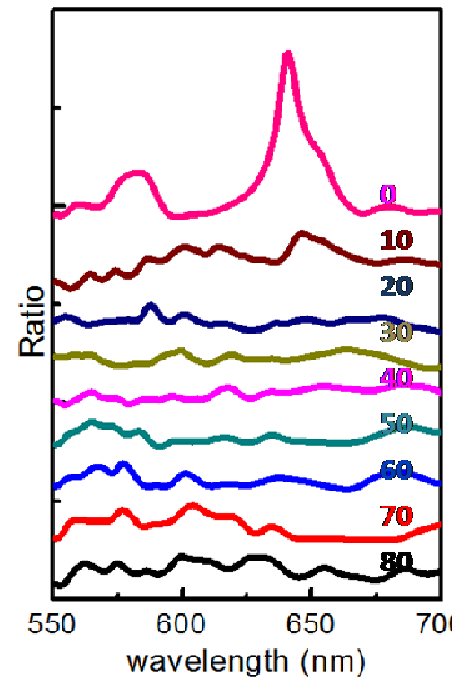

(b)

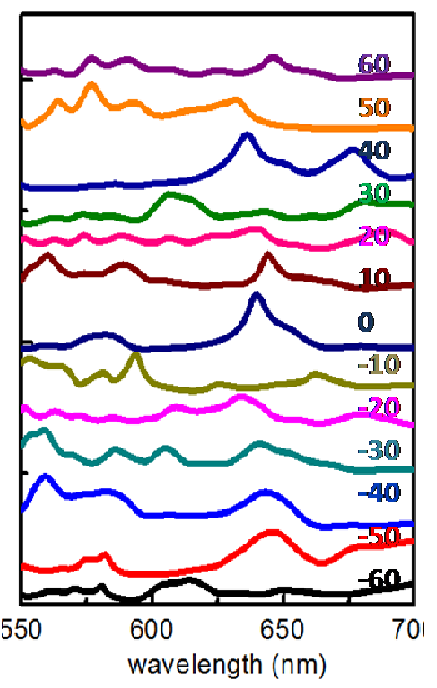

Figure S4. $(a, b)$ The spectra of the right to left ratio of electric field amplitude in the semi-annular apertures array with different incident angle. The incident angle is defined as the angle between the z-axis and the incident direction. (a) The incident light parallel to the $\mathrm{y}-\mathrm{z}$ plane, $\mathrm{kx}=0$. The incident angle changes from 0 to 80 degree. Both the two UL bands show sensitive dependence on the incident angle, and disappear when incident angle is larger than 10 degree. The oblique incidence in yz plane further damages the symmetry of the system, making it difficult to excite CSP mode. (b) The incident light parallel to the $x-z$ plane, $\mathbf{k}_{\mathrm{y}}=0$. The incident angle changes from -60 to 60 degree. The UL bands are also sensitive to the incident angle. In this case, the present symmetry keeps unchanged, so incident light can excite CSP mode at large incident angle and the UL can be found at large incident angle. Especially for the angle at \pm 40 degree, there are obvious UL bands near $630 \mathrm{~nm}$. 


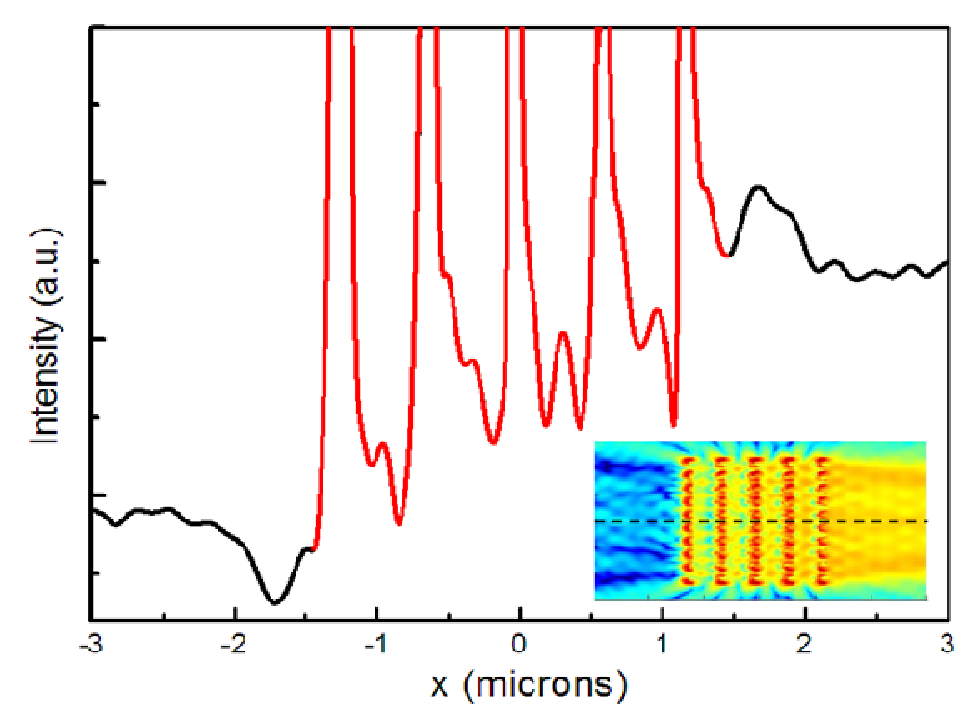

Figure S5. The FDTD simulated optical intensity cross section distribution of a five semi-annular aperture chain structure (along the line white line as sketched in inset). The red segment indicates the position of the structure. We find that the resonance intensity inside the apertures is step-increasing from left to right. This indicates that the unidirectional launched SPs wave in each chain have been stacked, thus amplifying the UL effect. 